\title{
HBA1 Gene
}

National Cancer Institute

\section{Source}

National Cancer Institute. HBA1 Gene. NCI Thesaurus. Code C75429.

This gene is involved in oxygen transport. 